\title{
On some early objections to Bohm's theory
}

\author{
WAYNE C. MYRVOLD \\ Department of Philosophy, University of Western Ontario, Canada
}

\begin{abstract}
Recent literature on Bohm's alternative to mainstream quantum mechanics may create the misleading impression that, except for perfunctory dismissals, the theory was ignored by the physics community in the years immediately following its proposal. As a matter of fact, Einstein, Pauli, and Heisenberg all published criticisms of Bohm's theory, explaining their reasons for not accepting the theory. These criticisms will be discussed and evaluated in this article.
\end{abstract}

\section{Introduction}

In 1952, David Bohm formulated a deterministic hidden-variable theory that supplements the quantum-mechanical state description with definite trajectories for particles, a theory that in many ways was anticipated by de Broglie's earlier pilot wave theory (de Broglie, 1928), which had been discussed at the 1927 Solvay congress. Bohm's theory did not meet with the acceptance in the physics community that Bohm had hoped for. It was not, however, ignored; several prominent physicists, among which were Einstein, Pauli, and Heisenberg, wrote articles expressing their reasons for not accepting Bohm's theory. These physicists regarded the discussion as a resumption of the discussion of de Broglie's theory that had taken place a quarter century earlier. After a brief introduction to Bohm's theory, these responses will be outlined in Section 3, below, and evaluated in the following sections. It seems to the present author that consideration of these early responses to Bohm's theory can serve as a useful backdrop to current controversies over the interpretation of quantum mechanics.

\section{Bohm's theory}

In his textbook, Quantum Theory (Bohm, 1951), David Bohm advocated a fairly orthodox version of the Copenhagen interpretation of quantum mechanics. The book is unusual, however, in the extent of the attention paid to the question of hidden variables. At several points in the course of the book the question is raised whether the probabilities appearing in quantum mechanics might arise as averages over supplementary variables appearing in a deeper, deterministic theory, beginning with $\$ 2.5$, "Unlikelihood of Completely Deterministic Laws on a Deeper Level". Bohm answers the question in the negative in $\$ 22.19$, "Proof that Quantum Theory is Inconsistent with Hidden Variables". 
It appears to be a dramatic change of viewpoint, therefore, when, shortly after Quantum Theory appeared, Bohm published what he had in his book declared to be impossible - an interpretation of quantum theory in terms of hidden variables (Bohm, 1952a, b). It has even been suggested (Olwell, 1999) that Bohm's shift from an orthodox viewpoint to a position somewhat removed from the mainstream opinion was prompted by the shift in his personal life, stemming from his dismissal from Princeton, from a position in the midst of a centre of physical research to a more marginal position in São Paolo, Brazil (see Peat, 1997 for an account).

A close look at the position outlined in Quantum Theory, however, reveals that the conceptual move from it to Bohm's hidden-variables theory is not as great as it initially appears. Bohm's proof in $\$ 22.19$ of the incompatibility of quantum theory with hidden variables refers the reader back to $\$ 6.11$, in which it is argued that "the wave aspects of matter are as real as are the particle aspects and, that, to obtain a compete and consistent theory, we must consider both aspects, each under its proper conditions" (Bohm, 1951, p. 136). Bohm here seems to presume that a hidden-variables theory will attempt to restore a picture of particles with definite positions and momenta, at the expense of the wave aspects of matter. An argument for the reality of these wave aspects counts, therefore, as an argument against hidden-variable theories.

In formulating his own hidden-variable theory, Bohm did not part with his view that the wave aspects of matter are as real as the particle aspects. On Bohm's theory, both the wave aspects and particulate aspects of matter are regarded as real. The wave function $\Psi$ is regarded as an actual physical field. Specification of the wave function, however, does not exhaustively specify reality according to this theory; there are, in addition, particles with definite trajectories, which depend on the wave function $\Psi$. The wave function is assumed to satisfy Schrödinger's equation. Furthermore, it is assumed that, at some time $t_{0}$, the probability density for finding a particle in a given region is given by the quantum-mechanical probability density

$$
\rho\left(\mathbf{x}, t_{0}\right)=\left|\Psi\left(\mathbf{x}, t_{0}\right)\right|^{2}
$$

and that the velocity of a particle located at point $\mathbf{x}$ at time $t_{0}$ is

$$
\mathbf{v}\left(\mathbf{x}, t_{0}\right)=-(\mathrm{i} \hbar / 2 m \rho)\left(\Psi^{\star} \nabla \Psi-\Psi \nabla \Psi^{\star}\right) .
$$

The acceleration of a particle is obtained by adding to the classical potential $V$ an additional "quantum potential", dependent on the wave function: ${ }^{1}$

$$
U=-\hbar^{2} \nabla^{2}|\Psi| / 2 m|\Psi|
$$

The assumption that the acceleration of a particle is given by $m \mathbf{a}=-\nabla(V+U)$, together with the assumption that the probability distribution for position and velocity satisfy the conditions (1) and (2) at some $t_{0}$, entails that the probability distribution will satisfy these conditions at all other times as well.

The orthodox, Copenhagen interpretation holds that any adequate theory must take into account both wave and particle aspects of matter. These aspects are held to be mutually exclusive; the same object cannot be both a particle and a wave simultaneously. Therefore, it is concluded, wave and particle concepts must be employed in a complementary manner, depending on the experimental situation. Bohm, in formulating his hidden-variables theory, accepts the premises of this argument but rejects the conclusion. While it is true that one object cannot be both a particle and a wave, two can; the Bohm theory employs particle and wave concepts simultaneously by applying them to different things. 
Bohm himself was, in his later writings, to emphasize the kinship between what he came to call the "ontological interpretation" and the Copenhagen interpretation. One of the most striking features of the Bohm theory is that, although it is assumed that an ideal position measurement will reveal the actual position of a particle, ${ }^{2}$ other physical quantities are contextualized; the result of an experiment is not due to properties of the measured system alone but depend crucially on the details of the experimental set-up. Thus, although each particle has a definite, differentiable trajectory, and so a definite velocity, hence momentum, at all times, this momentum is not, in general, the quantity that will be obtained via the sorts of procedures usually thought of as momentum measurements. Similarly, the result of what we usually regard as a spin measurement depends on the relative positions of the particle and the experimental apparatus, and cannot be regarded as an intrinsic property of the particle. In their book, Bohm and Hiley (1993) claim this context-dependence of experimental results to be an illustration of a central theme in Bohr's philosophy of quantum mechanics, namely, "the necessity of considering the whole experimental arrangement, the specification of which is imperative for any well-defined application of the quantum mechanical formalism" (Bohr, 1949, p. 230). Bohm and Hiley remark:

The context dependence of measurements is a further indication of how our interpretation does not imply a simple return to the basic principles of classical physics. It also embodies, in a certain sense, Bohr's notion of the indivisibility of the combined system of observing apparatus and observed object. Indeed it may be said that our approach provides a kind of intuitive understanding of what Bohr was saying. He described a 'measurement' as a whole phenomenon not further analyzable. The description of this phenomenon includes a specification of the experimental conditions (which are equivalent to the context that we have been discussing), along with a statement of the results. From the results and the conditions, one infers the 'values' of the 'measured' quantities, but as in our approach, it is not implied in Bohr's treatment that these values correspond to 'beables' that exist independently of the overall experimental context. (Bohm \& Hiley, 1993, pp. 122-123)

\section{Three responses: Einstein, Pauli, Heisenberg}

Bohm's theory did not meet with widespread acceptance. Despite the impression given in recent literature (see, e.g. Cushing, 1994; Peat, 1997; Olwell, 1999) the theory was not, however, ignored; several prominent physicists publicly expressed their reasons for not accepting the Bohm theory. In this section, we will discuss the criticisms of the Bohm theory levelled by Einstein (1953), Pauli (1952), and Heisenberg (1955, 1958).

\subsection{Einstein}

In an often-quoted letter to Max Born, Einstein remarked, "Have you noticed that Bohm believes (as de Broglie did, 25 years ago) that he is able to interpret the quantum theory in deterministic terms? That way seems too cheap to me" (letter of 12 May 1952, in Born, 1971, p. 192). In his contribution to the Born Festschrift volume (1953), Einstein expressed his reasons for finding the Bohm theory unsatisfactory. A reply from Bohm was included in the volume at Einstein's request.

In his article Einstein considered a simple, standard textbook example: a one-di- 
mensional particle in a box with purely elastic sides, i.e. a potential that is equal to 0 for $|x|<L / 2$, and is infinite otherwise. The energy eigenfunctions for such systems are equal to 0 outside the box $(|x|>L / 2)$, and inside the box are given by

$$
\psi_{n}(x, t)=\left\{\begin{array}{lc}
C e^{-\mathrm{i} E_{n} t / \hbar} \cos \left(k_{n} x\right), & n \text { odd } \\
C e^{-\mathrm{i} E_{n} t / \hbar} \sin \left(k_{n} x\right), & n \text { even }
\end{array}\right.
$$

where $k_{n}=n \pi / L, E_{n}=\hbar^{2} k_{n}^{2} / 2 m$, and $C=1 / \sqrt{2 L}$. Inside the box, $\psi_{n}$ is, therefore, an equally weighted superposition of a plane wave travelling to the right and a plane wave travelling to the left.

What does the Bohm theory say about a particle in a box, whose wave function is given by $\psi_{n}$ ? By the distribution postulate, at any given time $t$ the probability density for the location of such a particle is given by $\left|\psi_{n}(x, t)\right|^{2}$. Its velocity, however, is zero at all times, as can easily be verified by comparing expression (2) for the velocity of a Bohmian particle with the wave functions (4). Einstein finds this feature of the Bohm theory objectionable.

This objection, already raised against this theoretical attempt by Pauli a quarter century ago, is particularly serious with regard to our example. The vanishing of the velocity contradicts the well-founded requirement, that in the case of a macro-system the motion should agree approximately with the motion following from classical mechanics. ${ }^{3}$ (Einstein, 1953, p. 39)

In his reply to Einstein, Bohm pointed out, first of all, that his theory "is self-consistent and does not contradict any known experimental facts" (Bohm, 1953, p. 14). An analysis of the process of measurement leads to the conclusion that a process of the sort one usually regards as a measurement of momentum will, indeed, lead to one of the two results predicted by quantum mechanics, $\pm \hbar k_{n}$. Einstein's condition, that for macroscopic systems the theory yield an approximation, not only to the experimental predictions of classical mechanics, but also to the classical motions of unobserved particles, Bohm regards as an unwarranted demand:

In conclusion, the author would like to state that he would admit only two valid reasons for discarding a theory that explains a wide range of phenomena. One is that the theory is not internally consistent, and the second is that it disagrees with experiment. (Bohm, 1953, p. 18)

Although it is not clear whether he is doing so consciously, Bohm is here echoing Bohr's response to Einstein's criticisms of the Copenhagen interpretation:

In my opinion, there could be no other way to deem a logically consistent mathematical formalism as inadequate than by demonstrating the departure of its consequences from experience or by proving that its predictions did not exhaust the possibilities of observation. (Bohr, 1949, p. 228)

Thus, just as Bohr's insistence on taking the entire experimental arrangement into account serves Bohm as a justification for contextualizing certain quantities, Bohr's reply to Einstein's arguments serves Bohm's theory as a defence as well (or, perhaps, as poorly) as it serves the Copenhagen interpretation.

\subsection{Pauli}

Einstein regarded his criticism of the Bohm theory to be essentially the same as the 
criticism levelled by Pauli against de Broglie's pilot wave theory. It is not surprising, therefore, to find Pauli commenting on Bohm's theory in a collection of essays dedicated to de Broglie. In his essay, Pauli gives what he calls

the physical reasons, which have nothing to do with philosophical prejudices regarding the interpretation and justification of physical theories in general, that make me judge the interpretation of quantum mechanics based on the idea of complementarity to be the only admissible one. ${ }^{4}$ (Pauli, 1952, p. 42)

Pauli's reasoning is as follows. Either the "hidden" parameters have no observable consequences, or they lead to empirical predictions that differ from those of the quantum theory. In the former case, "the artificial asymmetry introduced in the treatment of the two variables of a canonically conjugate pair characterizes this form of the theory as artificial metaphysics" (p. 33). If, however, the new parameters are not "metaphysical" in nature, but give rise to empirically visible effects, these differences between particles regarded as identical will disrupt the symmetry or antisymmetry, respectively, of bosons or fermions with the same quantum numbers, and, so Pauli claims, lead to statistical predictions that, for systems composed of large numbers of "identical" particles, must differ appreciably from those of quantum statistical mechanics.

As Bohm's theory reproduces the empirical predictions of quantum mechanics, without making any new predictions, we will be concerned only with the first horn of Pauli's dilemma. Pauli's objection to the introduction of hidden variables that do not alter the statistical predictions of quantum mechanics is not a positivistic distrust of unobservable entities or of unmeasurable quantities. Rather, as he makes clear in his lectures on wave mechanics (Pauli, 1973) and in the 1956 preface to his Theory of Relativity (Pauli, 1958), the situation is regarded as analogous to the rejection of the aether by the special theory of relativity. The aether is to be rejected not merely because it is unobservable, but because it introduces an asymmetry-a distinguished rest frame-into the theory that is not present in the phenomena that serve as evidence for the theory. According to Pauli, Bohm's theory similarly introduces an "artificial asymmetry" in its treatment of position and momentum. In the standard formulation of quantum mechanics these canonically conjugate quantities are treated on a par. As Pauli points out in his lectures on wave mechanics (1973, p. 12), the basic formulas of the theory are invariant under the substitutions $x \leftrightarrow p, i \leftrightarrow-i$. For this reason, he says, it not necessary to treat either the position-space or momentum-space wave function as more fundamental than the other.

In Bohm's theory, on the other hand, position and momentum are not treated symmetrically. Although a measurement of position is assumed on Bohm's theory to yield the actual position of a particle, the theory eludes the von Neumann no-hiddenvariables-proof by "contextualizing" the momentum: the result of a the sort of experiment usually regarded as a measurement of momentum performed on a system $S$ is determined, not by the properties of $S$ alone, but also on the properties of the experimental apparatus, $A$.

As Bohm points out, the result of this interaction [between system and experimental apparatus] naturally depends also on the values of the parameters of $A$. What has been said here about position and momentum is valid generally for any pair of canonically conjugate variables: at most the values of one of these variables can be interpreted as a "property of $S$." This strips of its 
physical sense the simple passage via Fourier analysis from a wave function to a function of the conjugate variable (which leaves us the choice of considering either one or the other as the "primary" function), or introduces an asymmetry with regard to the interpretation of canonically conjugate magnitudes for which one finds reason neither in the system of our experiences nor in the mathematical formalism of wave mechanics. ${ }^{5}$ (Pauli, 1952, p. 39)

It is this asymmetry - the fact that the Bohm theory singles out position as the basic quantity whose statistical distribution is to be recovered, at the price of contextualizing momentum-rather than a general objection to determinism or to the positing of unobservable quantities that, in Pauli's opinion, makes Bohm's theory into "artificial metaphysics".

\subsection{Heisenberg}

Similar criticisms are found in Heisenberg's (1955, 1958) discussion of the Bohm theory. Like Pauli, Heisenberg distinguishes between counterproposals to the Copenhagen interpretation that have precisely the same empirical consequences as standard quantum mechanics, and those that do not. Bohm's theory lies in the former class; according to Heisenberg,

[t] he first consequence of this is that Bohm's interpretation cannot be refuted by experiment, and this is true of all the counter-proposals in the first group. From the fundamentally "positivistic" (it would perhaps be better to say "purely physical") standpoint, we are thus concerned not with counter-proposals to the Copenhagen interpretation, but with its exact repetition in a different language. (Heisenberg, 1955, p. 18; cf. 1958, pp. 129-130)

In declaring empirically equivalent theories to be nothing more than alternate formulations of the same theory, Heisenberg introduces an element of positivism absent from Pauli's and Einstein's discussions of the Bohm theory. One might think that Heisenberg could find little to object to in an "exact repetition" of the Copenhagen interpretation. His objection has to do with the suitability of the language used. The particle trajectories appearing in the Bohm theory, for Heisenberg, constitute "ideological superstructure" devoid of physical significance. As a further criticism, Heisenberg invokes Pauli's complaint of asymmetry.

Besides the objection already made that in speaking of particle orbits we are concerned with a superfluous "ideological superstructure," it must be particularly mentioned here that Bohm's language destroys the symmetry between position and velocity which is implicit in quantum theory; for the measurements of position Bohm accepts the usual interpretation, for the measurements of velocity or momentum he rejects it. Since the symmetry properties always constitute the most essential features of a theory, it is difficult to see what would be gained by omitting them in the corresponding language. Therefore, one cannot consider Bohm's counterproposal to the Copenhagen interpretation as an improvement. (Heisenberg, 1958, p. 133)

Heisenberg ends his discussion of criticisms of the Copenhagen interpretation with the remark,

All these proposals have found themselves compelled to sacrifice the essential 
symmetry properties of quantum theory (for instance, the symmetry between waves and particles or between position and velocity). Therefore, we may well suppose that the Copenhagen interpretation cannot be avoided if these symmetry properties-like the Lorentz invariance in the theory of relativity-are held to be a genuine feature of nature; and every experiment yet performed supports this view. (Heisenberg, 1958, pp. 145-146; cf. 1955, p. 28)

Einstein, Pauli, and Heisenberg, therefore, each object to the contextualization of momentum, Einstein because doing so violates the requirement that the theory pass over into a suitable classical limit, Pauli and Heisenberg because it violates the symmetries of quantum theory. Bohm himself took the asymmetry objection seriously. In his reply to Heisenberg's Physics and Philosophy, Bohm addressed the criticism by saying that, in objecting to this feature of the model, Heisenberg

shows that he perhaps did not appreciate that the only purpose of this phase of the work was to show that an alternative to the Copenhagen interpretation is at least logically possible. If one such interpretation is possible, then it is very likely that others will be possible too. Therefore, it should be reasonable to go on, using the original model as a starting point, with the aim of developing a formulation that is more plausible physically and elegant mathematically. (Bohm, 1962a, p. 270)

In another article published in the same year, Bohm re-emphasized the provisional nature of his proposal:

it should be kept in mind that before this proposal was made there had existed a widespread impression that no conceptions of hidden variables at all, not even if they were abstract, hypothetical, and "metaphysical," could possibly be consistent with the quantum theory ... it was therefore sufficient to propose any logically consistent theory that explained the quantum mechanics through hidden variables, no matter how abstract and "metaphysical" it might be. Thus, the existence of even a single consistent theory of this kind showed that whatever arguments one might continue to use against hidden variables, one could no longer use the argument that they are inconceivable. Of course, the specific theory that was proposed was not satisfactory for general physical reasons. But if one such theory is possible, then other and better theories may also be possible. And the natural implication of this argument is "Why not try to find them?" (Bohm, 1962b, p. 360, 1980, p. 81)

\section{Is the asymmetry necessary?}

If we take Bohm's suggestion seriously that his theory is to be regarded as a provisional one, showing that a "trajectory interpretation" 6 is logically possible, then it is worthwhile to explore alternatives. Pauli remarks that at most one of a pair of canonically conjugate variables can, in Bohm's theory, be regarded as a possessed property of the system; the other must be contextualized. There seems to be a suggestion that this is a necessary feature of theories like Bohm's; ascribing a definite value at all times to a dynamical variable comes only at the price of contextualizing its conjugate. In the spirit of Bohm's "Why not try to find them?" we should ask whether it is in fact possible for a theory to reproduce the statistical predictions of quantum mechanics while ascribing non-contextual values to both position and momentum. 
To get a feel for what such a theory might be like, consider the following special case. Consider a one-dimensional particle in a harmonic oscillator potential

$$
V(x)=\frac{1}{2} m \omega^{2} x^{2}
$$

As Wigner (1932, p. 752; see also Hillery et al., 1984, p. 137) has shown, for a harmonic oscillator, if the Wigner distribution

$$
w_{\psi}(x, p, t)=(1 / \pi \hbar) \int_{-\infty}^{\infty} \mathrm{d} y \psi^{\star}(x+y, t) \psi(x-y, t) e^{2 \mathrm{i} p y / \hbar}
$$

is non-negative at some time $t_{0}$, it remains so for all times, and in fact yields a distribution corresponding to an ensemble of harmonic oscillators. We have, therefore, in these special cases ensembles of trajectories such that the ensemble distribution of both position and momentum matches, at all times, the quantum-mechanical probability distributions for these quantities. Unfortunately, the Wigner distribution (or, rather, quasi-distribution), though always real-valued, can take on negative values, and so cannot be used as a basis for a comprehensive hidden-variable theory. Can an argument be given that no hidden-variable theory can ascribe non-contextual values to both position and momentum?

As Bell (1966) has emphasized, no-go theorems are not to be had for free; it is only when some conditions are specified that the hidden variables are assumed to satisfy that one obtains an impossibility result. Pauli's claim that at most one of position and momentum can be regarded as "possessed properties" is based on von Neumann's no-hidden-variables theorem. This theorem has rightly been criticized for imposing unreasonable constraints on simultaneously possessed values of observables that are not commeasurable (Bell, 1966; see Shimony, 1993, p. 89 for a report that Einstein earlier raised the same criticism). The Bell-Kochen-Specker theorem (Bell, 1966; Kochen \& Specker, 1967) weakens this constraint. The key assumption here is that, if $A$ and $B$ are observables whose corresponding operators commute, then the product observable $A B$ possesses a value that is the product of the values of $A$ and $B$. Hidden-variable theories satisfying this constraint are often called "non-contextual" hidden-variable theories, because the value associated with an observable is independent of the choice of which other commeasurable observables are to be measured. The Bell-Kochen-Specker theorem, while not ruling out hidden-variable theories tout court, does rule out theories that are non-contextual in this sense. The theorem leaves it open which subsets of observables can be ascribed non-contextual values, and in particular leaves it open whether the partial Boolean algebra generated by position and momentum is such a subset.

Rob Clifton (2000) has shown that it is possible to construct a Kochen-Speckerstyle obstruction involving position and momentum for a system having at least two continuous degrees of freedom. Clifton adapted Mermin's (1990, 1993) simplified proof of the Bell-Kochen-Specker theorem, itself based on work of Peres (1990), to show that it is not, in fact, possible to assign simultaneous non-contextual values to a pair of independent coordinates and their conjugate momenta. The following proof is based on Clifton's. ${ }^{7}$

Let $q_{1}, q_{2}$ be independent coordinates of some system, with conjugate momenta $p_{1}$, $p_{2}$, and let $\hat{q}_{1}, \hat{q}_{2}, \hat{p}_{1}, \hat{p}_{2}$ be the associated operators on the Hilbert space of the system. Define the operators 


$$
\begin{array}{ll}
\hat{A}_{1}=\cos \left(a \hat{q}_{1} / \hbar\right) & \hat{A}_{2}=\cos \left(a \hat{q}_{2} / \hbar\right) \\
\hat{B}_{1}=\cos \left(\pi \hat{p}_{1} / a\right) & \hat{B}_{2}=\cos \left(\pi \hat{p}_{2} / a\right)
\end{array}
$$

where $a$ is an arbitrary non-zero constant with dimensions of momentum. From the canonical commutation relations

$$
\begin{aligned}
& {\left[\hat{q}_{i}, \hat{q}_{j}\right]=\left[\hat{p}_{i}, \hat{p}_{j}\right]=0} \\
& {\left[\hat{q}_{i}, \hat{p}_{j}\right]=\mathrm{i} \hbar \delta_{i j} I}
\end{aligned}
$$

it follows that

$$
\left[\hat{A}_{1}, \hat{A}_{2}\right]=\left[\hat{B}_{1}, \hat{B}_{2}\right]=\left[\hat{A}_{1}, \hat{B}_{2}\right]=\left[\hat{B}_{1}, \hat{A}_{2}\right]=0
$$

and also that

$$
\begin{aligned}
& \hat{A}_{1} \hat{B}_{1}=-\hat{B}_{1} \hat{A}_{1} \\
& \hat{A}_{2} \hat{B}_{2}=-\hat{B}_{2} \hat{A}_{2} .
\end{aligned}
$$

We will assume that the quantities corresponding to $\hat{q}_{1}, \hat{q}_{2}, \hat{p}_{1}, \hat{p}_{2}$ take on definite values $q_{1}, q_{2}, p_{1}, p_{2}$, respectively, and that associated with the operators $\hat{A}_{1}, \hat{A}_{2}, \hat{B}_{1}, \hat{B}_{2}$ are the definite values

$$
\begin{array}{ll}
v\left(\hat{A}_{1}\right)=\cos \left(a q_{1} / \hbar\right) & v\left(\hat{B}_{1}\right)=\cos \left(\pi p_{1} / a\right) \\
v\left(\hat{A}_{2}\right)=\cos \left(a q_{2} / \hbar\right) & v\left(\hat{B}_{1}\right)=\cos \left(\pi p_{1} / a\right)
\end{array}
$$

These will be assumed to be all non-zero. We will also assume, as is usual in Kochen-Specker-type proofs, that if two operators $\hat{X}, \hat{Y}$ commute, and if the corresponding quantities take on definite values $v(\hat{X}), v(\hat{Y})$, then the product operator $\hat{X} \hat{Y}$ takes on a definite value $v(\hat{X} \hat{Y})=v(\hat{X}) v(\hat{Y})$.

Since $\hat{A}_{1}$ and $\hat{A}_{2}$ commute, the product operator $\hat{A}_{1} \hat{A}_{2}$ has definite value

$$
v\left(\hat{A}_{1} \hat{A}_{2}\right)=v\left(\hat{A}_{1}\right) v\left(\hat{A}_{2}\right) .
$$

Similarly,

$$
\begin{aligned}
v\left(\hat{B}_{1} \hat{B}_{2}\right) & =v\left(\hat{B}_{1}\right) v\left(\hat{B}_{2}\right) \\
v\left(\hat{A}_{1} \hat{B}_{2}\right) & =v\left(\hat{A}_{1}\right) v\left(\hat{B}_{2}\right) \\
v\left(\hat{A}_{2} \hat{B}_{1}\right) & =v\left(\hat{A}_{2}\right) v\left(\hat{B}_{1}\right) .
\end{aligned}
$$

Furthermore, the operator $\hat{A}_{1} \hat{A}_{2}$ commutes with $\hat{B}_{1} \hat{B}_{2}$, so

$$
v\left(\hat{A}_{1} \hat{A}_{2} \hat{B}_{1} \hat{B}_{2}\right)=v\left(\hat{A}_{1} \hat{A}_{2}\right) v\left(\hat{B}_{1} \hat{B}_{2}\right)=v\left(\hat{A}_{1}\right) v\left(\hat{A}_{2}\right) v\left(\hat{B}_{1}\right) v\left(\hat{B}_{2}\right) .
$$

Similarly, since $\hat{A}_{1} \hat{B}_{2}$ commutes with $\hat{A}_{2} \hat{B}_{1}$, we have

$$
\begin{gathered}
v\left(\hat{A}_{1} \hat{B}_{2} \hat{A}_{2} \hat{B}_{1}\right)=v\left(\hat{A}_{1} \hat{B}_{2}\right) v\left(\hat{A}_{2} \hat{B}_{1}\right)=v\left(\hat{A}_{1}\right) v\left(\hat{B}_{2}\right) v\left(\hat{A}_{2}\right) v\left(\hat{B}_{1}\right) \\
v\left(\hat{A}_{1} \hat{A}_{2} \hat{B}_{1} \hat{B}_{2}\right)=v\left(\hat{A}_{1} \hat{B}_{2} \hat{A}_{2} \hat{B}_{1}\right) .
\end{gathered}
$$

However, because of the anticommutation relations

$$
\hat{A}_{1} \hat{A}_{2} \hat{B}_{1} \hat{B}_{2}=-\hat{A}_{1} \hat{B}_{2} \hat{A}_{2} \hat{B}_{1}
$$

and therefore

$$
v\left(\hat{A}_{1} \hat{A}_{2} \hat{B}_{1} \hat{B}_{2}\right)=-v\left(\hat{A}_{1} \hat{B}_{2} \hat{A}_{2} \hat{B}_{1}\right)
$$

and thus we have a contradiction. 
This result shows that it is not possible for each of a pair of coordinates $q_{1}, q_{2}$ (which may pertain either to the same, or to different, particles) and their associated conjugate momenta to all have simultaneous non-contextual values. If we make the reasonable assumption that the issue of whether $q_{1}, p_{1}$ have non-contextual values is independent of whether $q_{2}, p_{2}$ have non-contextual values, and if we accept the assumption that possessed values of observables represented by commuting operators should satisfy the product rule, then we have a proof of Pauli's claim that, for any pair of canonically conjugate variables, at most the values of one of these variables can be interpreted as a property of the system. It can also be shown that, given a reasonable continuity condition on possessed values, the same result can be obtained while only considering a single degree of freedom - that is, a single coordinate and its conjugate momentum (Myrvold, 2002).

Clifton's argument depends on considerations of observables other than position and momentum; one could still ask whether some trajectory interpretation could yield ensemble distributions for position and momentum that match the quantum-mechanical probability distributions, without regard for other observables. Here, again, a no-go theorem is not to be had for free; one must specify some constraint on the trajectories. It turns out that there is a condition satisfied by the Bohm theory that cannot be satisfied by a theory that would seek to attribute definite trajectories to particles while avoiding the asymmetry of interpretation of which Pauli complains: unlike the Bohm theory, such a theory could not have dynamics that are local in configuration space.

The well-known non-locality exhibited by the Bohm theory stems from the fact that the wave function, for systems of $N>1$ particles, is not a function on 3-dimensional space but on $3 \mathrm{~N}$-dimensional configuration space. This means that, in general, the velocity of any one particle is a function of the coordinates of all $N$ particles, even when these are separated by a great distance. Only when the wave function factorizes is the motion of one particle independent of the position of the others. Bell (1980, p. 159, 1987, p. 115) has remarked,

That the guiding wave, in the general case, propagates not in ordinary three-space but in a multidimensional-configuration space is the origin of the notorious 'nonlocality' of quantum mechanics. It is a merit of the de BroglieBohm version to bring this out so explicitly that it cannot be ignored.

The Bohmian dynamics are, however, local in configuration space, and this means that, when the wave function is factorizable, so that each particle has its own wave function, the non-locality disappears. This feature, as we shall now show, entails the contextuality of momentum; a trajectory theory that attempts to attribute "possessed" values, revealed upon measurement, to both position and momentum cannot be local, even when only a single particle is involved.

Consider a single particle in one dimension. Let $\psi_{1}$ be a wave function that is non-zero only within a radius $r$ of the origin, and take $a \gg r$. Suppose that a particle has the wave function

$$
\Psi(x)=\left(\psi_{1}(x-a)-\psi_{1}(x+a)\right) / \sqrt{2} .
$$

The corresponding momentum-space wave function is given by

$$
\Phi(p)=\mathrm{i} \sqrt{2} \sin (p a / \hbar) \varphi_{1}(p)
$$

where the function $\varphi_{1}(p)$ is the Fourier transform of $\psi_{1}(x)$. Let us choose $\psi_{1}$ such that $\varphi_{1}$ is non-zero and indeed non-negligible at and near $p=0$. 
If we are to assign non-contextual values to both position and momentum for the particle whose wave function is given by (21), then the position of the particle must, with probability one, be in one of the disjoint regions $R^{+}=[a-r, a+r], R^{-}=[-a-r$, $-a+r$ ]. Since $\Phi(0)=0$, it cannot have zero momentum (and, indeed, for arbitrarily small $\varepsilon$ there will be an interval $\Delta$ containing $p=0$ such that the probability that $p \in \Delta$ is less than $\varepsilon$ ).

Now suppose that a particle-detection experiment is performed in $R^{+}$, with null result. We conclude that the particle is in $R^{-}$. But the probability density for a subsequent momentum "measurement" is $\left|\varphi_{1}(p)\right|^{2}$, which, by hypothesis, is non-negligible for $p=0$. Therefore, if we are to regard the momentum "measurements" as revealing pre-existing values of momentum, we must conclude that the particle-detection experiment performed in $R^{+}$must, at least some of the time, affect the momentum of the particle, even if the particle is located in $R^{-}$. Such a theory cannot, therefore, be local in configuration space, even when applied to a single particle.

\section{Should the criticism be taken seriously?}

Symmetry considerations are often regarded as extra-empirical, even aesthetic considerations. On such an attitude, the criticisms raised by Einstein, Pauli, and Heisenberg would count as extraordinarily weak, amounting, perhaps, to little more than an expression of distaste for Bohm's theory. This view, however, derives from an excessively narrow construal of evidential support, which locates the whole of the empirical support of a theory in its ability to save the phenomena. On this construal of the relation of empirical evidence to theories, any two predictively equivalent theories must be equally well supported by the evidence.

If, however, it is acknowledged that two theories having the same empirical consequences can be differentially supported by the evidence, a plausible principle is: ceteris paribus, a theory that posits asymmetries not present in the phenomena that serve as evidence for the theory is less well supported by the evidence than a theory whose symmetries match those of the phenomena. For example, if one postulates a preferred aether frame, which is nevertheless rendered undetectable by contraction of moving objects and slowing of physical processes in objects moving with respect to the aether, this precise cancellation of what would otherwise be observable effects appears as a remarkable coincidence. If one postulates however, the absence of an aether-or, at least, the absence of an aether with sufficient structure to permit it to pick out a preferred rest frame-together with the principle of relativity, the null result of experiments attempting to detect the motion of the earth with respect to the aether becomes inevitable. For these reasons, the invariance of experimental results with respect to choice of inertial reference frame lends support to Einstein's theory of relativity, and casts doubt on theories that posit that some inertial reference frame has a privileged status.

Can we advance a similar argument that the Bohm's theory's violation of $q / p$ symmetry detracts, not only from the elegance and beauty of the theory, but from its evidential support?

To begin addressing this question, let us first consider the role played by conjugate coordinate/momentum pairs in classical mechanics. In the Newtonian formulation of classical mechanics, the relation between position and momentum is a kinematical relation: 


$$
p_{i}=m \dot{q}_{i}
$$

Newton's second law then gives the rate of change of the momenta: ${ }^{8}$

$$
\dot{p}_{i}=-\partial V / \partial q_{i}
$$

In the Hamiltonian formulation of classical mechanics, position and momentum (or any other pair of canonically conjugate variables) are treated as if they are kinematically independent variables; the relation between position and momentum becomes one of Hamilton's equations of motion, into which (up to a change of sign) $p$ and $q$ enter symmetrically:

$$
\dot{q}_{i}=\partial H / \partial p_{i} \quad \dot{p}_{i}=-\partial H / \partial q_{i} .
$$

Because of this symmetric treatment of coordinates and their conjugate momenta, it is possible to make a canonical transformation that (again, up to a change in sign) interchanges the coordinates and momenta:

$$
Q_{i}=p_{i} \quad P_{i}=-q_{i}
$$

What are we to make of the invariance of the Hamiltonian equations of motion under such a transformation? Does the fact that Hamilton's equations of motion retain their form under the canonical transformation (26) indicate a physical symmetry?

The answer, I think, is no. Hamilton's equations of motion retain their form under arbitrary canonical transformations, no matter what form the Hamiltonian takes. The fact that Hamilton's equations of motion are invariant under the transformation (26), therefore, yields no information about the symmetries of a system. In this context, genuine physical symmetries are associated with a special class of canonical transformations-namely, those that leave the functional form of the Hamiltonian invariant. Such transformations have associated with them constants of the motion as their generating functions (see Goldstein, 1980, pp. 408-411 for a discussion).

There is, therefore, a profound disanalogy between the invariance of, say, the equations of electromagnetism under Lorentz transformations, and the invariance of the Hamiltonian equations of motion under the canonical transformation (26). One could say: the former reflects a symmetry of the physical system under consideration, the latter a symmetry of the phase space. The fact that structure of phase space does not distinguish between position and momentum should not be taken as an indication that there is no important physical distinction between position and momentum-and herein lies the disanalogy, because the fact that the spacetime, Einstein-Minkowski spacetime, invoked in special relativity is invariant under Lorentz transformations should be taken as an indication that, if the theory is correct, distinctions not preserved under Lorentz transformations do not belong to the structure of physical spacetime.

The situation is exactly analogous in quantum mechanics. Any unitary transformation

$$
\hat{q}_{i} \rightarrow \hat{U}^{\dagger} \hat{q}_{i} \hat{U} \quad \hat{p}_{i} \rightarrow \hat{U}^{\dagger} \hat{p}_{i} \hat{U}
$$

leaves the canonical commutation relations, and hence the equations of motion, invariant. There is, in particular, a unitary transformation that (up to a change of sign and multiplication by an arbitrary constant) interchanges position and momentum:

$$
\hat{q}_{i} \rightarrow \hat{p}_{i} / \alpha \quad \hat{p}_{i} \rightarrow-\alpha \hat{q}_{i}
$$

(Here $\alpha$ is an arbitrary constant of dimension mass/time). 
What are we to make of the invariance of the fundamental equations of quantum mechanics under such a transformation? Does this indicate a physical symmetry?

The answer, I think, is no. The canonical commutation relations are preserved under arbitrary unitary transformations (the analogue, in quantum mechanics, of classical canonical transformations), no matter what form the Hamiltonian takes. The fact that the fundamental equations of the theory are invariant under the transformation (28), therefore, yields no information about the symmetries of a system. In this context, a genuine physical symmetry is associated with a transformation represented by a unitary operator that commutes with the Hamiltonian.

The objection, as Heisenberg phrases it, is, therefore, too strong to be sustained. It is not true that a system subject to a transformation of the type (28), regarded as an active transformation, is experimentally indistinguishable from the untransformed system. Pace Heisenberg, the $q / p$ symmetry of the basic equations of quantum mechanics cannot be regarded as "a genuine feature of nature", nor can it be said that experiment supports this view.

Even if one rejects as excessively strong the assertion that symmetry under $q / p$ exchange constitutes an empirically observed symmetry comparable to Lorentz invariance, there remains a weaker objection that can be sustained. The fact that momentum measurements are not regarded as revealing the state of motion of the particle prior to observation introduces an element of arbitrariness into the Bohm theory. Bohm's theory makes a particular choice of possible trajectories of particles, not dictated by the requirement of recovering the quantum-mechanical predictions for position. Other choices are possible; among theories that share with Bohm's the attribution of trajectories to particles while recovering quantum-mechanical statistics for position are Nelson's stochastic theory (Nelson, 1966), the alternatives to the Bohm theory considered by Ghirardi and Deotto (1998), and Holland's "new trajectory interpretation" (Holland, 1998). These do not, of course, exhaust the logical possibilities. Although Bohm is correct that the experimental facts do not refute the contention that (for example) an electron in the ground state of a hydrogen atom is motionless, we can hardly be said to have evidence that it is motionless, evidence that would count against a rival trajectory interpretation that attributed a circulating orbit to such an electron. Any trajectory interpretation must make some choice regarding trajectories. The requirement that a trajectory theory recover the statistical predictions of quantum mechanics regarding position measurements radically underdetermines the trajectories of particles.

\section{The non-locality objection}

The discussions that followed the introduction of Bohm's theory are of historical interest, but they are also, I believe, of interest to present-day discussions regarding the foundations of quantum mechanics. Bohm's theory has the decided advantage that it solves the "measurement problem"-or, rather, in the Bohm theory the measurement problem does not arise, because there is no need to distinguish measurements from other interactions. The question arises whether the Bohm theory has disadvantages that should prompt us to try to find solutions to the measurement problem that lack them.

There is, of course, in addition to the $q / p$ asymmetry discussed above, another asymmetry exhibited by the Bohm theory. Its brand of non-locality requires a preferred notion of distant simultaneity. As Bohm and others have pointed out, it is possible for the theory to do so while maintaining Lorentz invariance at the phenomenal level. The 
objection to such a move is precisely the same as the objection to alternatives to special relativity that introduce an unobservable aether frame.

It is interesting that Pauli, Einstein, and Heisenberg did not raise the non-locality objection against Bohm's theory. Perhaps this is because Bohm's theory was introduced as an interpretation of non-relativistic quantum mechanics. Since then, however, this objection has been raised. There are three forms that a response to the objection can take. The first is much in the spirit of Bohm's reply to Einstein: the theory is logically consistent and makes the correct, Lorentz invariant predictions; in particular, as long as the distribution postulate is satisfied, it is not possible to gain sufficient knowledge of or control over initial conditions either to detect the preferred reference frame or to exploit the theory's non-locality for superluminal signalling. The second response is a $t u$ quoque objection; it may be claimed that Bell's theorem, together with the experimental tests of the Bell inequalities, shows that any empirically adequate theory must introduce a distinguished foliation at the fundamental level (this is suggested by Cushing, 1994, p. 189; see also Maudlin, 1996). The third is that every interpretation of quantum mechanics must pay the price of abandoning some cherished principle of pre-quantum physics, and that non-locality is a small price to pay for determinism and a resolution of the measurement problem.

The reply to the first form of response has already been discussed. Agreement with experimental results does not constitute the whole of the empirical support of a theory; ceteris paribus, a theory that does not introduce an asymmetry not present in the phenomena is better supported by those phenomena than one that does. Such a reply, of course, requires that the $t u$ quoque response fail. The violation of Bell's inequalities by quantum-mechanical predictions for entangled states is seen by some to indicate a fundamental incompatibility between quantum mechanics and relativity. Others have argued that the non-locality inherent in quantum mechanics is of a sort that can "peacefully coexist" with special relativity (see, e.g. Shimony, 1978, 1984, 1986; Jarrett, 1984, 1989; Butterfield, 1989; Redhead, 1989; Teller, 1989). Discussion of this issue is beyond the scope of the present article; we must content ourselves with the observation that, while Bohm's theory clearly does pick out a preferred notion of distant simultaneity, it is not at all clear that the violation of Einstein separability exhibited by ordinary quantum mechanics must do so also, and it is not clear that any exact formulation of quantum mechanics (that is, a formulation that does not invoke vague notions such as "measurement" or "observer") must pick out a preferred foliation or otherwise introduce asymmetries not present in the phenomena.

Here again, we should ask whether the asymmetry - the requirement of a preferred foliation for the formulation of the theory in spite of the Lorentz invariance of observable phenomena-is a feature inherent in the programme of constructing a trajectory interpretation, or merely a feature of Bohm's particular choice of trajectories. Berndl et al. (1996) have shown that it is not possible for a trajectory interpretation to yield a position distribution such that the quantum equilibrium condition $\rho=|\psi|^{2}$ holds with respect to all Lorentz frames. It is, however, possible to construct Lorentz-invariant Bohmian models by building a distinguished foliation into the model as one of the "beables" of the theory (Dürr et al., 1999). Although this issue deserves more discussion than can be given here, it seems that such a move is subject to precisely the same objection as the objection to alternatives to special relativity that introduce an unobservable aether frame-if the predictions of the theory matches those of quantum mechanics, the distinguished foliation will be unobservable and will introduce into the theoretical description an asymmetry not present in the phenomena. 
This leads to the third response - that some price must be paid, and that non-locality is a small price to pay for determinism and a satisfactory resolution to the measurement problem. Although it is not the primary motivation for interest in Bohmian theories, let us consider the issue of determinism first. If two predictively equivalent theories are equally well supported by the evidence, then choice between them - if a choice must be made at all — becomes a matter of personal taste, and those with a taste for determinism may indeed find non-locality a small price to pay. If, however, the methodological principle suggested above-that, ceteris paribus, a theory that does not introduce asymmetries not present in the phenomena is less well supported by those phenomena-is a reasonable one, then theories that, like the Bohm theory, introduce a preferred reference frame, are at an evidential disadvantage. We should ask whether this disadvantage is compensated for by the restoration of determinism. To ask this is to ask whether the evidence for determinism is sufficient to compensate for the evidential debit incurred by the introduction of a preferred reference frame. The answer to this question seems clearly to be negative; there is really nothing that counts as evidence that the world is deterministic at the fundamental level. Although we are, in many cases, able to predict events reliably, this predictability, as Schrödinger (1944) pointed out, is, even on a classical view of the world, typically a matter of order out of disorder-predictability depends on the statistical averaging of a myriad of microscopic events that are, at least for all practical purposes, effectively random.

This brings us to the matter of the measurement problem. It is indeed a problem for quantum mechanics, on the standard interpretation, that it is unable to account for the occurrence of determinate events at the macroscopic level and hence unable to account for the very measurement results that are taken as evidence for the theory. It is this problem that must be faced, if one is to claim a superiority of standard quantum mechanics over the Bohm theory.

\section{Acknowledgements}

The author would like to thank Andrew Wayne, Mélanie Frappier, James R. Brown, and two anonymous referees for helpful comments on an earlier draft of this article.

\section{Notes}

1. A variant on Bohm's theory known as "Bohmian mechanics" takes the law of motion to be given by (2); its acceleration is then a consequence of this law of motion. The difference between this formulation and Bohm's original formulation will be inessential for the discussion that follows.

2. It has been claimed (Englert et al., 1992) that the Bohm theory cannot sustain this assumption-that under certain circumstances, a particle detector will be "fooled" as to the position of a particle. Barrett (2000) has convincingly argued that this claim involves assumptions about the trajectory of particles in "free" space that are unwarranted in this context. A position measurement will always reveal the particle to be where the Bohm theory says it is, although other considerations might lead to different conclusions about the location of the particle.

3. Dieser übrigens schon vor einem Viertel-Jahrhundert von Pauli gegen diesen theoretischen Versuch erhobene Einwand ist im Hinblick auf unser Beispiel besonders schwerwiegend. Das Verschwinden der Geschwindigkeit widerspricht nämlich der wohlbegründeten Forderung, dass im Falle eines Makro-Systems die Bewegung mit der aus der klassischen Mechanik folgenden Bewegung angenähert übereinstimmen soll.

4. Ce sont ces raisons physiques, qui n’ont rien à faire avec des préjugés philosophiques quant à l'interprétation et à la justification de théories physiques en général, qui me font juger que l'interprétation de la mécanique quantique basée sur l'idée de la complémentarité est la seule admissible. 
5. Comme Bohm le fait remarquer, le résultat de cette interaction dépend naturellement aussi des valeurs des paramètres de $A$. Ce qui a été dit ici du lieu et de l'impulsion est valable d'une façon générale pour n'import quelle paire de variables canoniquement conjuguées: tout au plus les values d'une de ces variables peuvent être interprétées comme "propriété de S». Ceci dépouille de son sens physique le simple passage par analyse de Fourier d'une fonction d'onde à celle de la variable conjuguée (ce qui nous laisse le choix de considérer soit l'une soit l'autre comme la fonction "primaire») ou introduit une asymétrie par rapport à l'interpretation de grandeurs canoniquement conjuguées pour laquelle on ne trouve de raison ni dans le système de nos expériences ni dans le formalisme mathématique de la mécanique ondulatoire.

6. This apt phrase is Holland's (1998).

7. The difference between the Kochen-Specker obstruction given here and Clifton's is that Clifton's obstruction is not obtained directly for real-valued observables but via a detour through the complex plane; this is supplemented by an argument that an obstruction in the complex plane entails one on the real line.

8. In order to simplify the comparison between the Newtonian and Hamiltonian formulations of classical mechanics, we will restrict our attention to forces that can be derived from a potential — that is, monogenic forces (see Goldstein, 1980, p. 35).

\section{References}

BARrett, J. (2000) The persistence of memory: surreal trajectories in Bohm's theory, Philosophy of Science, 67, pp. 680-703.

BELL, J.S. (1966) On the problem of hidden variables in quantum mechanics, Reviews of Modern Physics, 38, pp. 447-452. Reprinted in Bell (1987, pp. 1-13).

BeLl, J.S. (1980) de Broglie-Bohm, delayed-choice double-slit experiment, and density matrix, International Fournal of Quantum Chemistry, 14, pp. 155-159.

Bell, J.S. (1987) Speakable and Unspeakable in Quantum Mechanics (Cambridge, Cambridge University Press).

Berndl, K., Dürr, D., Goldstein, S. \& Zanghì, N. (1996) Nonlocality, Lorentz invariance, and Bohmian quantum theory, Physical Review A, 53, pp. 2062-2073.

BoHm, D. (1951) Quantum Theory (New York, Prentice-Hall).

Boнm, D. (1952a) A suggested interpretation of the quantum theory in terms of 'hidden' variables, I, Physical Review, 85, pp. 166-179.

BoHm, D. (1952b) A suggested interpretation of the quantum theory in terms of 'hidden' variables, II, Physical Review, 85, pp. 180-193.

Boнm, D. (1953) A discussion of certain remarks by Einstein on Born's probability interpretation of the $\psi$-function, in: Scientific Papers Presented to Max Born (New York, Hafner), pp. 13-19.

BoHm, D. (1962a) Classical and non-classical concepts in the quantum theory: an answer to Heisenberg's Physics and Philosophy, British Fournal for the Philosophy of Science, 12, pp. 265-280.

BoHm, D. (1962b) Hidden variables in the quantum theory, in: D.R. BATES (Ed.) Quantum Theory III. Radiation and High Energy Physics (New York, Academic Press), pp. 345-387. Reprinted, with minor alterations, in: Wholeness and the Implicate Order (London, Routledge \& Kegan Paul, 1980), pp. 65-110.

Bohm, D. \& Hiley, B.J. (1993) The Undivided Universe (London, Routledge).

Bohr, N. (1949) Discussion with Einstein on epistemological problems in atomic physics, in: P.A. SCHILPP (Ed.) Albert Einstein: Philosopher-Scientist (La Salle, IL, Open Court), pp. 200-241.

BorN, M. (1971) The Born-Einstein Letters (New York, Macmillan).

Broglie, L. DE (1928) La nouvelle dynamique des quanta, in: Électrons et Photons: Rapports et Discussions du Cinquiéme Conseil de Physique (Paris, Gauthier-Villare et Cie.), pp. 105-132.

ButTerfield, J. (1989) A space-time approach to the Bell inequality, in: Cushing \& McMullin (Eds), pp. 114-144.

Clifton, R. (2000) Complementarity between position and momentum as a consequence of Kochen-Specker arguments, Physics Letters A, 271, pp. 1-7.

Cushing, J.T. (1994) Quantum Mechanics: Historical Contingency and the Copenhagen Hegemony (Chicago, IL, The University of Chicago Press).

Cushing, J.T. \& McMullin, E. (Eds) (1989) Philosophical Consequences of Quantum Theory: Reflections on Bell's Theorem (Notre Dame, IN, University of Notre Dame Press).

DürR, D., Goldstein, S., MünCh-Berndl, K. \& Zanghi, N. (1999) Hypersurface Bohm-Dirac models, Physical Review A, 60, pp. 2729-2736.

EInsteIN, A. (1953) Elementäre Überlegungen zur Interpretation der Grundlagen der Quanten-Mechanik, in: Scientific Papers Presented to Max Born (New York, Hafner), pp. 33-40. 
Englert, B.-G., Scully, M.O., Süssmann, G. \& Walther, H. (1992) Surrealistic Bohm trajectories, Zeitschrift für Naturforschung, 47a, pp. 1175-1186.

Ghirard, G.C. \& Deotro, E. (1998) Bohmian mechanics revisited, Foundations of Physics, 28, pp. 1-30. Goldstein, H. (1980) Classical Mechanics (Reading, MA, Addison-Wesley).

Heisenberg, W. (1955) The development of the interpretation of the quantum theory, in: W. PAULI (Ed.) Niels Bohr and the Development of Physics: Essays Dedicated to Niels Bohr on the Occasion of his Seventieth Birthday (New York, McGraw-Hill), pp. 12-29.

Heisenberg, W. (1958) Physics and Philosophy (New York, Harper \& Row).

Hillery, M., O’Connell, R.F., Scully, M.O. \& Wigner, E.P. (1984) Distribution functions in physics: fundamentals, Physics Reports, 106, pp. 121-167.

Holland, P.R. (1998) New trajectory interpretation of quantum mechanics, Foundations of Physics, 28, pp. 881-911.

JARRETT, J. (1984) On the physical significance of the locality conditions in the Bell arguments, Noûs, 18, pp. 569-589.

JARrett, J. (1989) Bell's theorem: a guide to the implications, in: Cushing \& MCMullin (Eds), pp. 60-79.

Kochen, S. \& Specker, E.P. (1967) The problem of hidden variables in quantum mechanics, fournal of Mathematics and Mechanics, 17, pp. 59-87.

Maudlin, T. (1996) Space-time in the quantum world, in: J.T. Cushing, A. Fine \& S. Goldstein (Eds) Bohmian Mechanics and Quantum Theory: An Appraisal (Dordrecht, Kluwer Academic), pp. 285-307.

Mermin, N.D. (1990) Simple unified form for the major no-hidden-variables theorems, Physical Review Letters, 65, pp. 3373-3377.

Mermin, N.D. (1993) Hidden variables and the two theorems of John Bell, Reviews of Modern Physics, 65, pp. 803-815.

MYRVOLD, W.C. (2002) Kochen-Specker $\varepsilon$-obstruction for position and momentum using a single degree of freedom, Physics Letters A, 299, pp. 8-14.

Nelson, E. (1966) Derivation of the Schrödinger equation from Newtonian mechanics, Physical Review, 150, pp. 1079-1085.

Olwell, R. (1999) Physical isolation and marginalization in physics: David Bohm's Cold War exile, Isis, 90, pp. $738-756$.

PAUli, W. (1952) Remarques sur le problème des paramètres cachés dans la mécanique quantique et sur la théorie de l'onde pilote, in: Louis de Broglie: Physicien et Penseur (Paris, Éditions Albin Michel), pp. 33-42.

Pauli, W. (1958) Theory of Relativity (New York, Pergamon Press). Reprint: New York, Dover, 1981.

Pauli, W. (1973) Wave Mechanics. Pauli Lectures on Physics, Vol. 5, C.P. Enz (Ed.), S. Margulies \& H.R. LEwIS (Trans.) (Cambridge, MA, The MIT Press). Reprint: New York, Dover, 2000.

PeAt, F.D. (1997) Infinite Potential: The Life and Times of David Bohm (Reading, MA, Addison-Wesley).

Peres, A. (1990) Incompatible results of quantum measurements, Physics Letters A, 151, pp. 107-108.

RedHeAd, M.L.G. (1989) Incompleteness, Nonlocality, and Realism: A Prolegomenon to the Philosophy of Quantum Mechanics (Oxford, Oxford University Press).

SChröDInger, E. (1944) What is Life? The Physical Aspect of the Living Cell (Cambridge, Cambridge University Press).

Shimony, A. (1978) Metaphysical problems in the foundations of quantum mechanics, International Philosophical Quarterly, 18, pp. 3-17.

Shimony, A. (1984) Controllable and uncontrollable non-locality, in: S. KAMEFUCHI et al. (Eds) Foundations of Quantum Mechanics in Light of New Technology (Tokyo, The Physical Society of Japan), pp. 225-230. Reprinted in SHIMONY (1993, pp. 130-138).

Shimony, A. (1986) Events and processes in the quantum world, in: R. Penrose \& C. Isham (Eds) Quantum Concepts in Space and Time (Oxford, Oxford University Press), pp. 182-203. Reprinted in SHIMONY (1993, pp. 140-162).

Shimony, A. (1993) Search for a Naturalistic World View, Volume II: Natural Science and Metaphysics (Cambridge, Cambridge University Press).

Teller, P. (1989) Relativity, relational holism, and the bell inequalities, in: CushING \& MCMulLIN (Eds), pp. 208-223.

Wigner, E. (1932) On the quantum correction for thermodynamic equilibrium, Physical Review, 40, pp. 749-759. 


\section{W. C. MYRVOLD}

\section{Note on contributor}

Wayne C. Myrvold is Assistant Professor in the Department of Philosophy at the University of Western Ontario. He is the author of a number of articles on the interpretation of quantum mechanics, including "On peaceful coexistence: is the collapse postulate compatible with relativity?", Studies in History and Philosophy of Modern Physics, 33 (September 2002), pp. 435-466, and "Relativistic quantum becoming", forthcoming in The British Fournal for the Philosophy of Science. Correspondence: Department of Philosophy, University of Western Ontario, London, Ontario, Canada N6A 3K7. E-mail: wmyrvold@uwo.ca 